\title{
Snapshot Generation in a Constructive Object-Oriented Modeling Language
}

\author{
Mauro Ferrari ${ }^{1}$, Camillo Fiorentini $^{2}$, Alberto Momigliano ${ }^{2}$, and Mario Ornaghi ${ }^{2}$ \\ ${ }^{1}$ Dipartimento di Informatica e Comunicazione, Università degli Studi dell'Insubria, Italy \\ mauro.ferrari@uninsubria.it \\ ${ }^{2}$ Dipartimento di Scienze dell'Informazione, Università degli Studi di Milano, Italy \\ \{fiorenti, momiglia, ornaghi\}@dsi.unimi.it
}

\begin{abstract}
CooML is an object-oriented modeling language where specifications are theories in a constructive logic designed to handle incomplete information. In this logic we view snapshots as a formal counterpart of object populations, which are associated with specifications via the constructive interpretation of logical connectives. In this paper, we introduce the "snapshot semantics" of CooML and we describe a snapshot generation (SG) algorithm, which can be applied to validate specifications in the spirit of OCL-like constraints over UML models. Differently from the latter and from the standard BHK semantics, the logic allows us to exploit a notion of partial validation that is appropriate to encodings characterised by incomplete information. SG is akin to model generation in answer set programming. We show that the algorithm is sound and complete so that its successful termination implies consistency of the system.
\end{abstract}

\section{Introduction}

We are developing the constructive object-oriented modeling language CooML [19] (http: / / cooml.dsi.unimi.it), a specification language for OO systems. Similarly to UML/OCL [23], CooML provides a framework for the design of system specifications in the early stages of the development process. The language allows the user to distinguish between internally defined elements and the problem domain, which may involve loosely or incompletely defined components. This encourages the selection of the appropriate level of abstraction w.r.t. specifications.

CooML follows the spirit of lightweight formal methods [10]: it does not focus on full formalization, nor on whole system correctness, but emphasizes partiality in analysis and specification. In particular, in the context of OO modeling, both the validation of a specification and the check of its consistency can be achieved via the notion of snapshot, i.e. a population of objects in a given system state that satisfies the specification. Previous work has used snapshots for validation of UML/OCL models [8] and specifications in JML [4].

The novelty of CooML's approach resides in its semantics, which is related to the constructive explanation of logical connectives (a.k.a. the BHK interpretation [22]). Specifically, the truth of a CooML proposition in a given interpretation is explained by a mathematical object that we call an information term. For the time being, the latter can be visualized as a sort of proof term inhabiting a type/formula. The underlying logic is 
characterized by how classical and constructive information co-exists, the main "entry" point being the different way in which an atomic formula $A$ is given evidence (for more details we refer the kind reader to the original formulation of the logic in [15]). If we call the pair $I: P$ a piece of information, where $P$ is a formula and $I$ is its information term, then $I: P$ may be true or false in a classical interpretation $w$, called a world. Thus, we have a notion of a model of a piece of information based on classical logic. In particular, we use $\mathrm{T}\{F\}$ to indicate the truth of $F$; in fact, $\mathrm{T}$ does not contain evidence for $F$, but it yields a trivial piece of information true in all the models of $F$. This introduces a novel and flexible way to handle incomplete information, a notorious difficulty in information systems such as relational databases.

Crucially, the constructive side of the logic allows the identification of snapshots with information terms, thus providing a formal counterpart to the intuitive notion of object populations. We argue that CooML's proof-theoretic snapshot generation may be advantageous in comparison to a model-theoretic one, especially in cases where not all the information required to define a model is even present. The possibility of treating information in this less committed way means that we can select only the relevant information; this may have a cascade of benefits in terms of conciseness of the representation.

The contributions of this paper are twofold. First, we extend the semantics developed in purely logical terms in [15] to object oriented modeling languages. We regard an OO system specification as a CooML theory $T$, the system snapshots as the pieces of information $I: T$, and the related information content as a suitable set of formulae. We show that the latter can be seen as the minimum information needed to give evidence to snapshots and we relate that to snapshot consistency. Secondly, we describe (and implement) a snapshot generation algorithm (SGA), taking as inputs: (i) a CooML theory $T$, axiomatizing a set of classes in a problem domain PD; (ii) the user's generation requirements $\mathscr{G}$ - they serve an analogous purpose of domain predicates in the grounding phase of ASP's [17]. As snapshots should be consistent with respect to PD and $\mathscr{G}$, we prove that consistency checking is sound and that snapshot generation is complete, i.e. if a consistent snapshot satisfying the generation requirements exists, it will be generated. This is loosely connected to adequacy results in the theory of CLP's [7].

\section{CooML Specifications}

In this section we informally present the language via an example (adapted from [3]), while we defer the formal exposition to Section 2.1. The problem domain concerns a small coach company. Each coach has a specified number of seats and can be used for regular or private trips. In a regular trip, each passenger has its own ticket and seat number. In a private trip, the whole coach is rented and there may be a guide. The corresponding CooML specification is contained in the package coachCompany (Fig. 1). To explain our example we need to introduce CooML types system. We distinguish among data types (in our example, Integer and Boolean), PD types (Person), and object types (Coach, Trip, Passenger). They all inherit from the top type Value the identity relation and the string representation. Data types are "statically" defined, i.e., their values do not depend on the current state. CooML assumes the 


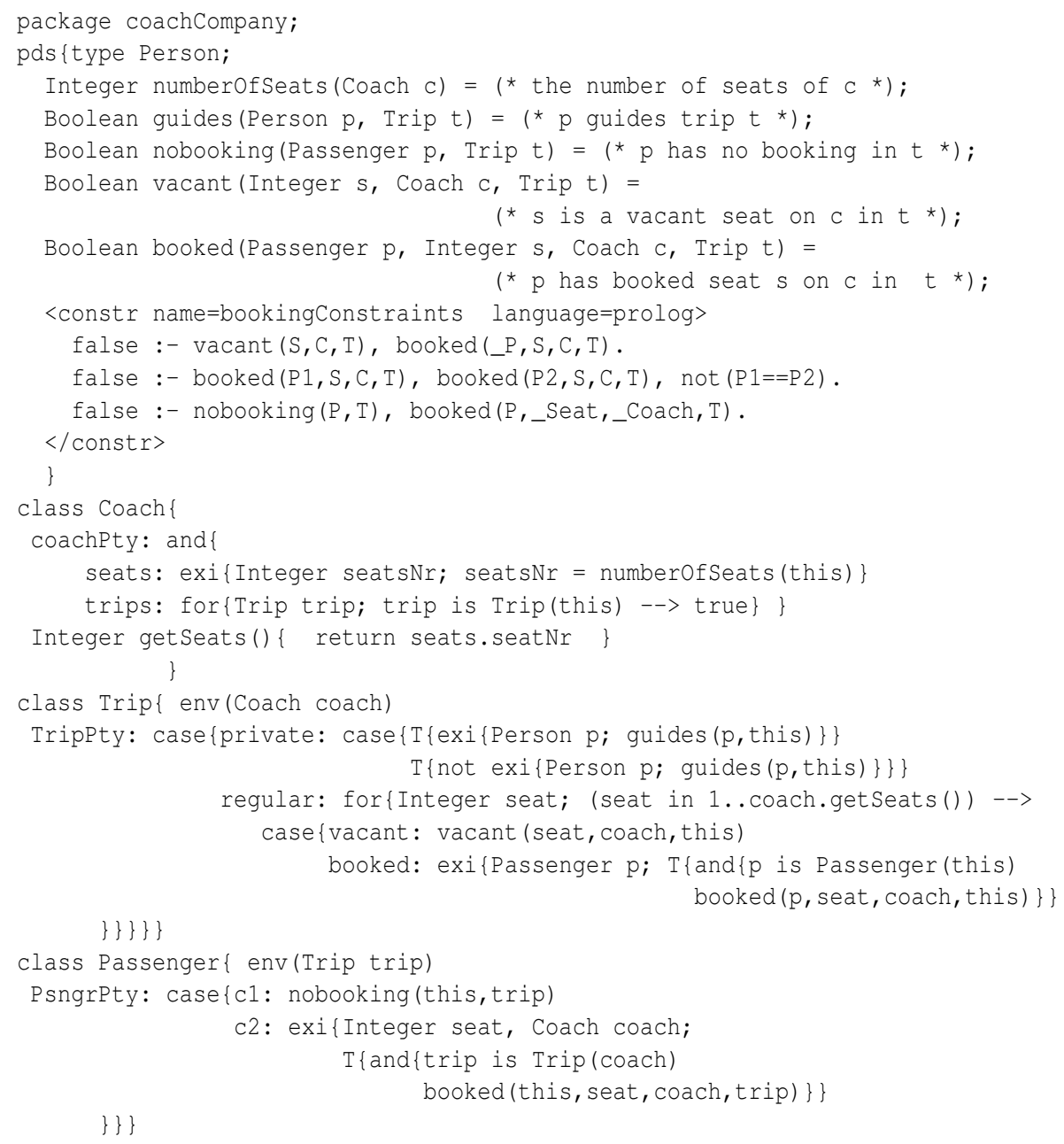

Fig. 1. The coachCompany package

existence of an implementation that evaluates ground terms to values. A PD type extends Value with a set of problem domain functions.

Nothing is assumed about PD types; they may be characterized by a set of formal or loose properties that we call $P D$ constraints, introduced by the tag <constr $>$.

The special subtype Obj of Value introduces object identities. Objects are created by CooML classes, which are structured in a single inheritance hierarchy rooted in Obj. The definition of a class $C$ may depend on some environment parameters; namely $\mathrm{C}(\underline{e})$ is a class with environment parameters $\underline{e}$. If $\underline{\mathbf{e}}$ is a ground instance of the environment parameters $\underline{e}$, then $C(\underline{\mathbf{e}})$ can be used to create new objects. We write "o is C ( $\underline{\mathbf{e}}$ )" to indicate that $\mathbf{o}$ has been created by $\mathrm{C}(\underline{\mathbf{e}})$, while "o instanceof $\mathrm{C}(\underline{\mathbf{e}})$ " means that o has environment $\underline{\mathbf{e}}$ and has been created by a subclass $\mathrm{C}$ ' of $\mathrm{C}$. We call those class predicates. 
In a package: (i) data types are assumed to be externally implemented; (ii) PD types are defined in the pds (problem domain specification) section; (iii) classes are introduced by suitable class declarations.

pds declaration and world states. The pds section specifies our general knowledge of the problem domain. It introduces PD types, functions and predicates using data and class types. In our example we introduce the PD type Person and functions numberOfSeats, guides, ... The informal descriptions $(* \ldots *)$ use terms of the global signature provided by the analysis phase [11]. A $<$ constr $>$ declaration introduces a set of PD constraints representing general problem domain properties that are not interpreted by CooML, but possibly by some external tool. In the example, PD constraints are expressed in Prolog assisting the SG algorithm in filtering out undesired snapshots. The class predicate " $\mathrm{O}$ is $\mathrm{C}(\underline{e})$ " is represented by the Pro$\log$ predicate $i \mathrm{sOf}(\mathrm{O}, \mathrm{C},[\underline{e}])$, while "o istanceOf $\mathrm{C}(\underline{e})$ " is translated into instanceOf $(\mathrm{O}, \mathrm{C},[\underline{e}])$. The first constraint says that a coach seat cannot be vacant and booked at the same time, the second one excludes overbooking (a seat can be booked by at most one person), while the third says that the predicate nobooking $(P, T)$ holds if person $\mathrm{P}$ has not booked a seat on the coach associated with trip $\mathrm{T}$. In this paper, we assume that the signature $\Sigma_{T}$ of a CooML theory $T$ (including PD types, data types and classes) is first order and that we can represent the possible states of the "real world" by reachable $\Sigma_{T}$-interpretations, dubbed world states. Reachability means that each element of the interpretation domains is represented by some ground terms, in our case CooML values. In a world state, PD symbols are interpreted over the external world, data types are interpreted according to their implementation, and class predicates represent the current system objects. For instance the class predicates

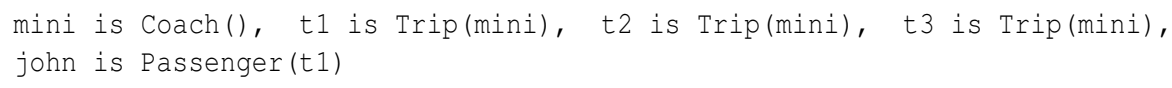

represent a small company with a single mini-bus mini, three trips $t 1, t 2, t 3$ operated by mini and, so far, only one passenger john associated with trip $t 1$.

class declarations and properties. A class declaration introduces the name $C$ of the class, its (possible) environment parameters $\underline{e}$, its property $\operatorname{Pt} y_{C}\left(\operatorname{thhis}_{\underline{\mathrm{s}}}\right)$, and its methods 1 . An object $\mathbf{0}$ created by $\mathrm{C}(\underline{\mathbf{e}})$ stores a piece of information structured according to $\operatorname{Pt} y_{C}(\mathbf{o}, \underline{\mathbf{e}})$, and uses the methods implemented by $\mathrm{C}(\underline{\mathbf{e}})$.

For class properties, CooML uses a prefix syntax, where formulas may be labeled. Labels are used to refer to subformulae. For example, the label seats is used in the getseats method to refer to seatsNr. A class property $P$ is an atomic formula over $\Sigma_{T}$, or (recursively) a formula of the form and $\left\{P_{1} \ldots P_{n}\right\}$, case $\left\{P_{1} \ldots P_{n}\right\}$, exi $\{\underline{\tau} \underline{x} ; P\}$, for $\{\underline{\tau} \underline{x} ; G \rightarrow P\}$ and $\mathrm{T}\left\{P^{e x t}\right\}$, where $P^{e x t}$ is a property that may also use negation not and implication imp. We stress that not and imp cannot be used outside $\mathrm{T}$.

In CooML's semantics, a property $P$ defines a set of possible pieces of information of the form $I: P$, where $I$ is an information term, that is a structure justifying the truth of $P$. Each piece of information $I: P$ has an information content, i.e. a set of simple properties intuitively representing the minimum amount of information needed to justify $P$ according to $I$. A simple property is either an atom or of the form $\mathrm{T}\left\{P^{e x t}\right\}$. A simple property $S$ represents a basic information unit, i.e., it has a unique information term $t$ t

\footnotetext{
${ }^{1}$ We use the self-reference this as in Java.
} 
where $t t$ is a constant. This means that the only information we have is the truth of $S$, and that the associated information content is simply the set $\{S\}$. Exemplifying,

$$
\text { tt : } t 1 \text { is Trip(mini) }
$$

has information content $\{t 1$ is $\operatorname{Trip}(\operatorname{mini})\}$ and means that the trip $t 1$ is assigned to the coach mini in the current world state.

The operator $\mathrm{T}$ may enclose a complex property $P$ and indicates that we are interested only in its truth. Let us consider

$$
t t: T\{\text { exi Person } p ; \text { guides }(p, t 2)\}\} \quad t t: T\{\text { not exi\{Person } p ; \text { guides }(p, t 3)\}\}
$$

The first piece of information says that $t 2$ is a guided trip without indicating who the guide is; the second one says that $t 3$ has no guide.

By default 2 the truth of a simple property $S$ in a world state w (denoted w $\models S$ ) is defined as in classical logic, by ignoring $\mathrm{T}$ (i.e., $\mathrm{w} \models \mathrm{T}\{P\}$ iff $\mathrm{w} \models P$ ) and interpreting case as $\vee$, and as $\wedge$, not as $\neg$, imp as $\rightarrow$, exi as $\exists$ and $\operatorname{for}\{\underline{\tau} \underline{x} ; G(\underline{x}) \rightarrow P(\underline{x})\}$ as $\forall \underline{x}(G(\underline{x}) \rightarrow P(\underline{x}))$.

In contrast, non-simple properties are interpreted constructively, by means of information terms. A piece of information $I: P$ may have one of the following forms:

Existential. $(\mathbf{x}, I)$ : exi $\{\tau x ; P(x)\}$, where $\tau$ is the type of the existential variable $x$. The term $\mathbf{x}$ is a witness for $x$ and the information content is the one of $I: P(\mathbf{x})$. For example,

$$
(4, t t): \operatorname{exi}\{\text { Integer seatNr; seatNr }=\text { numberofSeats }(\operatorname{mini})\}
$$

has witness 4 and information content $\{4=$ numberofSeats (mini) $\}$, signifying that our mini-bus has 4 passenger seats. Note that, differently from the case of simple properties, we know the value of $x$ that makes $P(x)$ true.

Universal. $\left(\left(\mathbf{x}_{1}, I_{1}\right), \ldots,\left(\mathbf{x}_{n}, I_{n}\right)\right)$ : for $\{\tau x ; G(x) \rightarrow P(x)\}$, where $G(x)$ is an $x$-generator, i.e. a formula true for finitely many $x 3$. The information content is the union of those of $I_{1}: P\left(\mathbf{x}_{1}\right), \ldots, I_{n}: P\left(\mathbf{x}_{n}\right)$ and of the domain property dom $\left(x ; G(x) ;\left[\mathbf{x}_{1}, \ldots, \mathbf{x}_{n}\right]\right)$, a special simple property interpreted as $\forall x\left(G(x) \leftrightarrow \operatorname{member}\left(x,\left[\mathbf{x}_{1}, \ldots, \mathbf{x}_{n}\right]\right)\right)$. For example, the information content of

$$
((t 1, t t),(t 2, t t),(t 3, t t)): \text { for }\{\operatorname{Trip} t r i p ; \text { trip is } \operatorname{Trip}(\min i) \rightarrow \text { true }\}
$$

is $\{$ dom(trip; trip is Trip(mini); [t1,t2,t3])\}, showing that the domain of the trip-generator "trip is Trip (mini)" is $\{t 1, t 2, t 3\}$. Since the atomic formula true corresponds to no information, it can be ignored.

Conjunctive. $\left(I_{1}, \ldots, I_{n}\right)$ : and $\left\{P_{1} \ldots P_{n}\right\}$. The information content is the union of those of $I_{j}: P_{j}$, for all $j \in 1$..n. For instance, a piece of information for the class property coachPty (mini) and the related information content $I C_{1}$ is

$$
\begin{gathered}
((4, t t),((t 1, t t),(t 2, t t),(t 3, t t))): \text { and }\{\text { seats (mini) trips (mini) }\} \\
\left.I C_{1}=\{4=\text { numberofSeats (mini), dom(trip; trip is Trip(mini); }[t 1, t 2, t 3])\right\}
\end{gathered}
$$

\footnotetext{
${ }^{2}$ But one can change this, although we do not discuss it for lack of space.

${ }^{3} \mathrm{We}$ omit here the precise syntax of generators.
} 
Disjunctive. $\left(\mathrm{k}, I_{k}\right)$ : case $\left\{P_{1} \ldots P_{n}\right\}$. The selector $k \in 1 . . n$ points to the true subformula $P_{k}$ and the information content is $I_{k}: P_{k}$ 's. For example, if the object john with class predicate john is Passenger(t1) contains the information term $(1, t t)$, then

$$
(1, t t): \operatorname{case}\{c 1 \text { :nobooking }(j \circ h n, t 1) c 2: \ldots\}
$$

selects the first sub-property of PsngrPty, with information content \{nobooking ( john, t1) \}, i.e. john has no booking in trip t 1 in the current state.

The information content of classes. Let $C(\underline{e})$ be a class with property $\operatorname{Pty}_{C}($ this, $\underline{e})$. We associate with $C$ the class axiom

$$
\text { clAx }(\mathrm{C}): \operatorname{for}\{\text { Obj this, } \underline{\tau} \underline{e} \text {; this is } \mathrm{C}(\underline{e}) \rightarrow \operatorname{Pty}(\operatorname{this}, \underline{e})\}
$$

The corresponding pieces of information and information content are those for universal properties. The piece of information for class Coach and its information content $I C_{2}$ is:

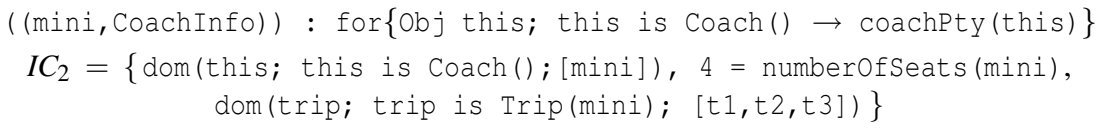

where CoachInfo: coachPty (mini) is defined as in the conjunctive case.

System snapshots and their information content. Let $P$ be a package introducing a set of constraints $\mathscr{T}$ and the CooML classes $C_{1}, \ldots, C_{n}$. We associate with $P$ a CooML theory $T_{P}=\langle\operatorname{thAx}, \mathscr{T}\rangle$, where thAx $=\operatorname{and}\left\{\mathrm{clAx}\left(C_{1}\right) \cdots \mathrm{clAx}\left(C_{n}\right)\right\}$.

A piece of information $I$ : thAx represents the information content of the whole system. We call it a system snapshot, to emphasise that the system may evolve through a sequence $I_{0}$ : thAx $, \ldots, I_{n}$ : thAx, ... A snapshot for our coachCompany system is of the form:

$$
\left(I_{1}, I_{2}, I_{3}\right): \operatorname{and}\{\mathrm{clAx}(\mathrm{Coach}) \mathrm{clAx}(\text { Passenger) clAx (Trip) }\}
$$

and possible information terms $I_{1}, I_{2}, I_{3}$ are

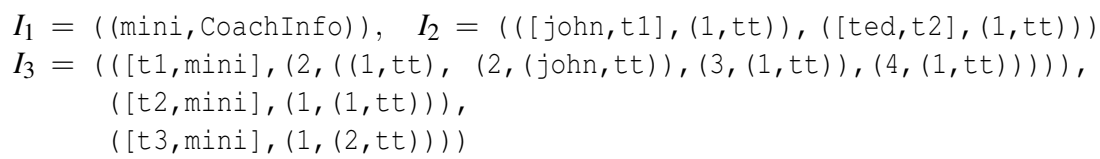

where [...] denote tuples. A relevant part of the information content for coachCompany is given in Fig. 2.

The above information content could be seen as an "incompletely specified" model of the coachCompany theory, where numberofSeats, nobooking, vacant, booked and class predicates are completely specified, while for guides we have only some partial knowledge, expressed by the T-properties, and moreover nothing is said about Person. The relationship with classical models can be better explained by comparing the constructive and classical reading of CooML properties. 


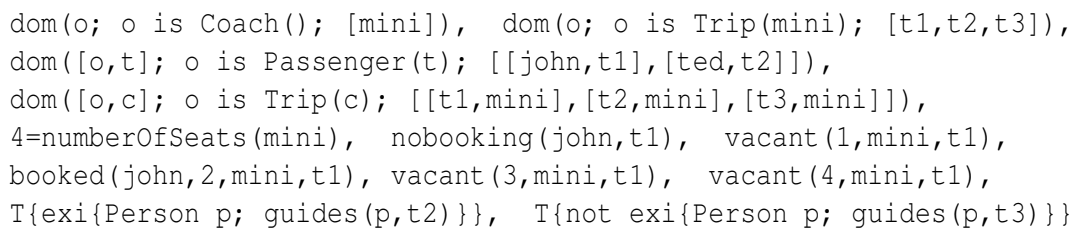

Fig. 2. Part of the information content of coachCompany

Let $T=\langle$ thAx, $\mathscr{T}\rangle$ be a CooML theory. We can switch to the classical interpretation of thAx simply by using the $\mathrm{T}$ operator, i.e. by considering the simple property $\mathrm{T}\{$ thAx $\}$. One can prove that $\mathrm{T}\{\mathrm{thAx}\}$ has a reachable model if and only if so does $\operatorname{IC}(I: \operatorname{thAx})$, for at least one piece of information $I:$ thAx. Furthermore, one can prove that $\operatorname{IC}(I: \operatorname{thAx})$ is the minimum set of simple formulas that justifies $I$ as an explanation of thAx.

In this context we are mainly interested in the notion of consistency with respect to the PD constraints, assuming that the latter can be interpreted as first order sentences. In our example, we interpret a program clause $H:-B_{1}, \ldots, B_{n}$ as the universal closure of $B_{1} \wedge \ldots \wedge B_{n} \rightarrow H$, as usual. A system snapshot $I:$ thAx for a theory $T=\langle$ thAx, $\mathscr{T}\rangle$ is consistent if its information content $\operatorname{IC}(I: \operatorname{thAx})$ is true in a reachable classical model of $\mathscr{T}$; $T$ is consistent if there is a consistent snapshot for it. For example, the above snapshot $\left(I_{1}, I_{2}, I_{3}\right)$ is consistent with respect to the first and second constraint of the pds section, but not with the third, since both nobooking (john, t1) and booked (john, 2, mini, t1) belong to the information content of coachCompany (Fig. 2).

\subsection{Formal Definitions}

Let $T=\langle\operatorname{thAx}, \mathscr{T}\rangle$ be a CooML theory and $\Sigma_{T}$ the associated first order signature. The set of information terms for a property $P, \operatorname{IT}(P)$, is inductively defined as follows, where $\underline{\mathrm{x}}$ stands for values of $\underline{x}$ of the appropriate type:

$$
\begin{array}{ll}
\operatorname{IT}(P) & =\{t t\}, \text { if } P \text { is simple } \\
\operatorname{IT}\left(\operatorname{and}\left\{P_{1} \cdots P_{n}\right\}\right) & =\left\{\left(I_{1}, \ldots, I_{n}\right) \mid I_{j} \in \operatorname{IT}\left(P_{j}\right) \text { for all } j \in 1 . . n\right\} \\
\operatorname{IT}\left(\operatorname{case}\left\{P_{1} \cdots P_{n}\right\}\right) & =\left\{(k, I) \mid 1 \leq k \leq n \text { and } I \in \operatorname{IT}\left(P_{k}\right)\right\} \\
\operatorname{IT}(\operatorname{exi}\{\underline{x} \underline{x} ; P\}) & =\{(\underline{\mathbf{x}}, I) \mid I \in \operatorname{IT}(P)\} \\
\operatorname{IT}(\operatorname{for}\{\underline{\underline{x}} ; G(\underline{x}) \rightarrow P\}) & =\left\{\left(\left(\underline{\mathbf{x}}_{1}, I_{1}\right), \ldots,\left(\underline{\mathbf{x}}_{n}, I_{n}\right)\right) \mid I_{j} \in \operatorname{IT}(P) \text { for all } j \in 1 . . n\right\}
\end{array}
$$

A piece of information for a closed property $P$ is a pair $I: P$, with $I \in \operatorname{IT}(P)$. A collection is a set of closed simple properties. The information content $\operatorname{IC}(I: P)$ is the collection inductively defined as follows:

$$
\begin{array}{ll}
\operatorname{IC}(\text { tt }: P) & =\{P\}, \text { where } P \text { is a simple property } \\
\operatorname{IC}\left(\left(I_{1}, \ldots, I_{n}\right): \operatorname{and}\left\{P_{1} \ldots P_{n}\right\}\right) & =\bigcup_{j=1}^{n} \operatorname{IC}\left(I_{j}: P_{j}\right) \\
\operatorname{IC}\left((k, I): \operatorname{case}\left\{P_{1} \ldots P_{n}\right\}\right) & =\operatorname{IC}\left(I: P_{k}\right) \\
\operatorname{IC}((\underline{\mathbf{x}}, I): \operatorname{exi}\{\underline{\tau} \underline{x} ; P(\underline{x})\}) & =\operatorname{IC}(I: P(\underline{\mathbf{x}})) \\
\operatorname{IC}\left(\left(\left(\underline{\mathbf{x}}_{1}, I_{1}\right), \ldots,\left(\underline{\mathbf{x}}_{n}, I_{n}\right)\right): \operatorname{for}\{\underline{\tau} \underline{x} ; G(\underline{x}) \rightarrow P(\underline{x})\}\right)= & \bigcup j=1 \\
& \cup\left\{\operatorname{IC}\left(I_{j}: P\left(\underline{\mathbf{x}}_{j}\right)\right)\right.
\end{array}
$$


The information content IC $(I: P)$ represents the minimum amount of information needed to get evidence for $P$ according to $I$. We say that a collection $\mathscr{C}$ gives evidence to $I: P$, and we write $\mathscr{C} \triangleright I: P$, iff one of the following clauses holds:

$$
\begin{aligned}
& \mathscr{C} \triangleright \text { tt : } P \\
& \mathscr{C} \triangleright\left(I_{1}, \ldots, I_{n}\right): \operatorname{and}\left\{P_{1} \ldots P_{n}\right\} \quad \text { iff } \mathscr{C} \triangleright I_{j}: P_{j} \text { for all } j \in 1 . . n \\
& \mathscr{C} \triangleright(k, I): \operatorname{case}\left\{P_{1} \ldots P_{n}\right\} \quad \text { iff } \mathscr{C} \triangleright I: P_{k} \\
& \mathscr{C} \triangleright(\underline{\mathbf{x}}, I): \operatorname{exi}\{\underline{\tau} \underline{x} ; P(\underline{x})\} \quad \text { iff } \mathscr{C} \triangleright I: P(\underline{\mathbf{x}}) \\
& \mathscr{C} \triangleright\left(\left(\underline{\mathbf{x}}_{1}, I_{1}\right), \ldots,\left(\underline{\mathbf{x}}_{n}, I_{n}\right)\right): \text { for }\{\tau \underline{x} ; G(\underline{x}) \rightarrow P(\underline{x})\} \text { iff } \operatorname{dom}\left(\underline{x} ; G(\underline{x}) ;\left[\underline{\mathbf{x}}_{1}, \ldots, \underline{\mathbf{x}}_{n}\right]\right) \in \mathscr{C} \\
& \text { and } \mathscr{C} \triangleright I_{j}: P\left(\underline{\mathbf{x}}_{j}\right) \text { for all } j \in 1 . . n
\end{aligned}
$$

The information content IC $(I: P)$ represents an information about the current world state. We define the information content of $\mathscr{C}$ as its closure under (classical) logical consequence, for $\mathscr{C}^{*}=\{P|\mathscr{C}|=P\}$. We say that $\mathscr{C}_{1}$ contains less information than $\mathscr{C}_{2}$ (written $\mathscr{C}_{1} \sqsubseteq \mathscr{C}_{2}$ ) iff $\mathscr{C}_{1}^{*} \subseteq \mathscr{C}_{2}^{*}$. Intuitively, the definition of $\sqsubseteq$ is justified by the fact that an user will "trust" $\mathscr{C}^{*}$, whenever he trusts $\mathscr{C}$. We could use a different trust-relation, considering different logics. We only need this to hold:

(1). $\mathscr{C} \subseteq \mathscr{C}^{*}$

(2). $\mathscr{C}_{1} \subseteq \mathscr{C}_{2}^{*}$ implies $\mathscr{C}_{1} \sqsubseteq \mathscr{C}_{2}$.

Using the above properties, we can establishes the minimality of $\operatorname{IC}(I: P)$ with respect to $\sqsubseteq$ :

Theorem 1. Let $I: P$ be a piece of information:

1. $\operatorname{IC}(I: P) \triangleright I: P$

2. For every collection $\mathscr{C}, \mathscr{C} \triangleright I: P$ implies $\operatorname{IC}(I: P) \sqsubseteq \mathscr{C}$.

Now we can apply the above discussion to the problem of checking snapshots against constraints. Let $T=\langle\operatorname{thAx}, \mathscr{T}\rangle$ be a CooML theory. We recall that a snapshot for $T$ is a piece of information $I$ : thAx. We introduce two notions of consistency for snapshots.

- A snapshot $I:$ thAx is consistent with respect to the constraints $\mathscr{T}$ ( $\mathscr{T}$-consistent) iff there exists a reachable model of $\operatorname{IC}(I: \operatorname{thAx}) \cup \mathscr{T}$.

- $T$ is snapshot-consistent iff there is at least one snapshot $I$ : thAx such that $I$ : thAx is $\mathscr{T}$-consistent.

The latter definition is related to classical consistency by the following result:

Theorem 2. Let $T=\langle t h A x, \mathscr{T}\rangle$ be a CooML theory. $T$ is snapshot-consistent iff there is a reachable model of $\mathrm{T}\{t h A \mathrm{x}\} \cup \mathscr{T}$.

\section{A Snapshots Generation Algorithm and Its Theory}

A snapshot generation algorithm (SGA) for a CooML theory $T=\langle$ thAx, $\mathscr{T}\rangle$ takes as input the user's generation requirements and tries to produce $\mathscr{T}$-consistent snapshots that satisfy such requirements. Roughly, generation states represent incomplete 
snapshots, i.e. in logic programming parlance, partially instantiated terms; inconsistent attempts are pruned, when recognized as such during generation.

Consistency checking plays a central role. It depends on the PD logic and it is discussed next. In Subsection 3.2 we illustrate the use of snapshot generation for validating CooML specifications. Finally, in Subsection 3.3 we briefly outline a non deterministic algorithm based on which one may develop sound and complete implementations.

\subsection{Consistency Checking}

Here we briefly discuss a simplified version of consistency checking in our Prolog implementation, called SnaC. To recognize inconsistent attempts, $S G A$ uses an internal representation of the information content of the current generation state $S$, denoted by INFO $_{S}$. Let $P_{S}$ be the internal Prolog translation of the information content $\mathrm{INFO}_{S}$. For this simplified version, we assume that $P_{S}$ is executed by a suitable meta-interpreter. Without giving the formal details, we notice that $\mathrm{INFO}_{S}$ consists either of ground facts, clauses of the form $H$ :- eq $(t 1, t 2)$ or false :- $B$, where:

- We use eq to avoid Prolog's standard unification interfering with Skolem constants. Indeed, the latter represent unknown values originating from the translation of $\mathrm{T}\{\operatorname{exi}\{\ldots\}\}$, where different constants may represent the same value. In this simplified account, the eq atoms are just residuated by the meta-interpreter in a list of "unsolved equations".

- The reserved atom false is adopted to detect inconsistency: its finite failure signals snapshot consistency, conversely, its success corresponds to inconsistency.

Clauses whose head isfalse are called integrity constraints and false may occur only as such. A SnaC representation $P_{S}$ has the following property: if the metainterpretation of a goal $G$ succeeds from $P_{S}$ with answer $\sigma$ and a list $L$ of unsolved equations, then $G \sigma$ is a logical consequence of $P_{S} \cup L$. Furthermore, consistency is preserved and the models of $P_{S}$ are models of $\mathrm{INFO}_{S}$ (in the declarative reading of $P_{S}$, where we interpret eq as equality and false as falsehood). As an example, let us consider the SnaC representation $P_{c C o m p}$ in Fig. 3 of the information content of the coachCompany package (Fig. 2).

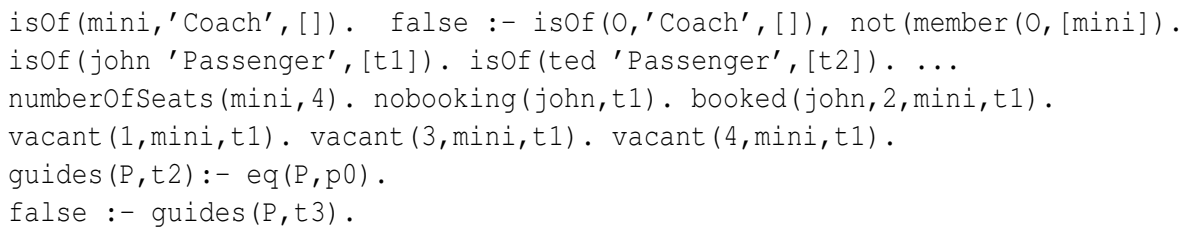

Fig. 3. The SnaC representation $P_{c C o m p}$

The facts and the constraint in the first lines come from the translation of domain properties. For example, the first row contains the translation of $\operatorname{dom}\{0 ; 0$ is $\operatorname{Coach}() ;[\operatorname{mini}])\}$. The other facts come from the translation of atoms. The clause guides $(P, t 2):-e q(P, p 0)$ is the translation of 
$\mathrm{T}\{\operatorname{exi}\{$ Person $\mathrm{p} ;$ guides $(\mathrm{p}, \mathrm{t} 2)\}\}$, where $\mathrm{p} 0$ is a fresh Skolem constant. Finally, false :- guides $(P, t 3)$ is the translation of $T$ \{not exi\{Person $p$; guides $(\mathrm{p}, \mathrm{t} 3)\}$ \}.

Let us analyse the three possible outcomes of consistency checking starting from the example in Fig. 3:

(a) false finitely fails from the program $P_{c C o m p}$. This entails that false does not belong to the minimum model $\mathscr{M}$ of $P_{c C o m p} \cup\{$ eq $(\mathrm{X}, \mathrm{X})\}$. The latter contains all the ground atoms in Fig. 3 as well as guides (p0, t2 ). Since $\mathscr{M}$ is a model of $P_{c \text { Comp }}$, it is also a model of the information content of the coachCompany package thanks to the properties of the translation.

(b) If we add to $P_{c \text { Comp }}$ the constraint

C1) false :- nobooking $(P, T)$, booked $(P, S S, C C, T)$.

now the goal false succeeds from program $P_{c C o m p} \cup\{c 1\}$, residuating the empty list. This implies that the snapshot corresponding to the information content of coachCompany is inconsistent w.r.t. $\mathrm{c} 1$.

(c) If we instead add the constraint

C2) false :- guides $(\mathrm{P}, \mathrm{T})$, isof (P,'Passenger', [T] ).

the goal false succeeds from program $P_{c C o m p} \cup\{\mathrm{c} 2\}$, residuating [eq (ted,p0)]. This implies that false belongs to the minimum model $\mathscr{M}$ of $P_{c C o m p} \cup\{\mathrm{c} 2$, eq ( ted, $\left.\mathrm{p} 0)\right\}$. The equality eq ( $t e d, \mathrm{p} 0$ ) is returned to the user as a source of inconsistency.

The above discussion is reflected in the following theorem:

Theorem 3. Let $T=\langle t h A x, \mathscr{T}\rangle$ be a CooML theory, $I$ : thAx a snapshot and $P$ a program containing the translation of $\mathrm{IC}(I: t h A x)$ and of the PD constraints $\mathscr{T}$.

1. If fal se finitely fails from $P$, then $I:$ thAx is $\mathscr{T}$-consistent.

2. If false succeeds from $P$ residuating a set of constraints $\mathscr{U}$, then $I: t h A x$ is inconsistent with respect to $\mathscr{T} \cup \mathscr{U}$.

In the first case, SnaC accepts $I$ : thAx as a $\mathscr{T}$-consistent snapshot. In the second, $\mathscr{U}$ being empty signals inconsistency. If $\mathscr{U}$ is not empty, it is returned as an answer.

A more general result can be established admitting a larger class of simple properties and PD constraints, via techniques similar to those used in CLP, such as constraint systems [7]. Roughly, we can consider $\mathscr{T}$ as a program of a CLP system whose calculus is an extension of the standard logic programming operational semantics and where the constraint system is the Herbrand universe under CET, modified to deal with Skolem constants.

\subsection{Validating Specifications Via SG}

One of the purposes of snapshot generation is understanding and validating a CooML specification. To this aim, the user can specify suitable generation requirements in order 
to reduce the number of generated examples to a manageable size and show only the aspects he is interested in. We explain the language of generation requirements and its semantics through our example. It may be helpful to keep in mind the analogy with the behaviour of an answer set program during grounding.

In the implementation, the number of generated snapshots can be limited by means of the the special atom choice $(A)$. This plays the role of domain predicates in ASP. The SG algorithm will instantiate $A$ according to its axiomatisation. For example:

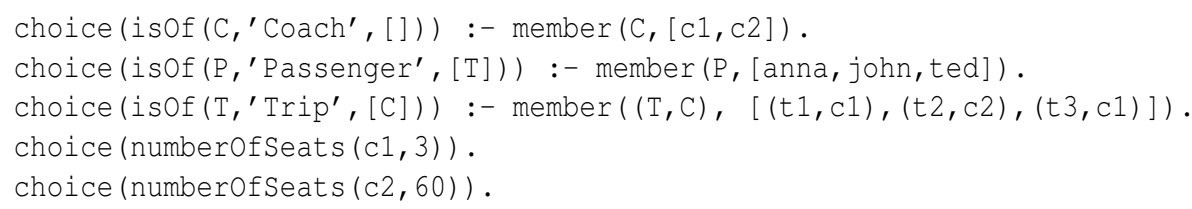

instructs SG to generate one coach $\mathrm{c} 1$ with 3 seats and possible trips $t 1$, t3, and another $c 2$ with 60 seats and trip $t 2$. The declarative meaning of choice is given by the axiom schema $A \rightarrow$ choice $(A)$, which, together with the user definition of choice, sets up the generation requirements. The generated snapshots will satisfy the PD constraints, as well as the generation requirements.

Once the SG algorithm loads a CooML theory and the user generation requirements, it can be queried with generation goals ( $G$-goals). A sample $G$-goal is:

$$
\text { (g1) [ [3,tt], Trips ] : isOf (C,'Coach', [] ). }
$$

Since $[3, t t]$ : seats $(C)$ has information content $3=$ numberOfSeats $(C)$, the query looks for the information Trips: trips (C) for every coach C with 3 seats. More precisely, the $G$-goal includes both a generation goal ("generate all the coaches C with 3 seats that satisfy the generation requirements") and a query ("for each $\mathrm{C}$, show the information on the trips assigned to it"). An answer to $\mathrm{g} 1$ is:

$$
\text { Trips }=[[t 1, t t]] \text { and } C=c 1
$$

with information content

$$
\text { isof (c1,' 'Coach', []), isof (t1, 'Trip', [c1]) }
$$

The rest of the snapshot, including information terms for all classes in the package, is omitted for the sake of space. If the user asks for more solutions, all possible snapshots will be shown. In the above example, there are two more solutions, where $\mathrm{c} 1$ has two trip assigned or none.

We now sketch some ways in which SG can be used in the process of system specification and development. This will be the focus of future work.

Validating specifications. The goal here is to show that a CooML theory "correctly" models the problem domain. Validation is empirical by nature: it relates the theory to the modeled world. The idea is to generate models that satisfy given generation requirements and check whether they match the user expectations. To this aim, it is useful to tune the generation requirements to consider separately various aspects that can be understood within a small, "human viable" number of examples, as usual in this context [8]. For instance, we may concentrate on the validation of the booking part of the 
CoachCompany package. In particular, we can find some supporting evidence of the correctness of the specification in a match between the expected and actual number of snapshots, where parameters of the latter are chosen as small as possible, while preserving meaningfulness. Naturally, snapshots can be used as inputs to tools for automatic, specification-based testing generation, in the spirit of [18].

Partial and full model checking. As traditional in software model checking, here the goal is to show that, under the assumptions of the generation requirements, no snapshot satisfies an undesired property. This is obtained if the SGA finds a snapshotinconsistency, i.e. it halts without exhibiting any snapshot. Equivalently, one can prove that every snapshot satisfies a given property by showing that its negation is snapshotinconsistent. We call this approach partial model checking, because in general snapshot consistency may depend on the selection of generation requirements. We may perform full model checking if the set of generated snapshots is representative of all models of the theory w.r.t. the property under consideration.

\subsection{A Schematic Algorithm}

We now describe a general schema for the snapshot generation algorithm, of which SnaC is just a first rough implementation. Let $T=\langle$ thAx, $\mathscr{T}\rangle$ be a CooML theory, where thAx $=$ and $\left\{\mathrm{clAx}\left(C_{1}\right), \ldots, \mathrm{clAx}\left(C_{n}\right)\right\}$. Its information terms are represented by sets of $G$-goals that we call populations. The generation process starts from a set $P_{0}$ of $G$-goals to be solved, i.e. to become ground. The SGA gradually instantiates $P_{0}$, possibly generating new $G$-goals. It divides the population in two separate sets: ToDo, containing the $G$-goals not solved yet and DoNE, containing the solved ones. A generation state has the form $S=\langle$ Done, ToDo, CLosed, INFO $\rangle$, where:

- Closed is a set of predicates $\operatorname{closed}(C, \underline{e})$, which is extended when all the objects with creation class $C(\underline{e})$ have been generated. It prevents the creation of new objects of class $C(\underline{e})$ in subsequent steps.

- INFO is the representation in the PD language of the information content of DONE, i.e. for every $I$ : isof $(o, C,[\underline{e}]) \in \operatorname{Done}, \operatorname{IC}\left(I: P t y_{C}(o, \underline{e})\right) \subseteq \operatorname{InFO}$.

The following definitions are in order:

- A state $S$ is in solved form if ToDo $=\emptyset$.

- $\operatorname{Dom}(S)=\{i \operatorname{isof}(o, C,[\underline{e}]) \mid I: \operatorname{isOf}(o, C,[\underline{e}]) \in \operatorname{Done} \cup$ ToDo $\}$.

- $S_{1} \preceq S_{2}$ for $S_{i}=\left\langle\mathrm{DONE}_{i}, \mathrm{ToDO}_{i}, \mathrm{CLOSED}_{i}, \mathrm{INFO}_{i}\right\rangle$ iff

1. $\operatorname{DoNE}_{1} \subseteq \operatorname{DonE}_{2}, \operatorname{Dom}\left(S_{1}\right) \subseteq \operatorname{Dom}\left(S_{2}\right)$ and $\mathrm{INFO}_{1} \subseteq \mathrm{INFO}_{2}$;

2. If $\operatorname{closed}(C, \underline{e}) \in \mathrm{CLOSED}_{1}$, then isof $(o, C,[\underline{e}]) \in \operatorname{Dom}\left(S_{1}\right)$ iff isof $(o, C,[\underline{e}]) \in \operatorname{Dom}\left(S_{2}\right)$.

The SGA starts from initial state $S_{0}=\langle\emptyset$, ToDo $0, \emptyset, \emptyset\rangle$ and yields a solution state $S=\langle$ Done, $\emptyset$, Closed, INFO $\rangle$ such that $S_{0} \preceq S$; since ToDo $=\emptyset$, for every $I: i \operatorname{sof}(o, C,[\underline{e}]) \in \operatorname{ToDo}_{0}$, Done contains a ground information term $(I$ : isof $(o, C,[\underline{e}])) \sigma$ solving it. The algorithm computes a solution of $S_{0}$ that is minimal with respect to $\preceq$ through a sequence of expansion steps. The latter are triples $\left\langle S, I: \operatorname{isOf}(o, C,[\underline{e}]), S^{\prime}\right\rangle$ such that: 
p1. $I$ : isOf $(o, C,[\underline{e}]) \in$ ToDo (the selected goal);

p2. $(I: \operatorname{isOf}(o, C,[\underline{e}])) \sigma \in \operatorname{DonE}^{\prime}$ and $I: \operatorname{isOf}(o, C,[\underline{e}]) \notin \operatorname{ToDo}^{\prime}$ (it has been solved);

p3. $S \prec S^{\prime}$ and, for every $S^{*}$ in solved form, $S \prec S^{*} \preceq S^{\prime}$ entails $S^{*}=S^{\prime}$ (no solution is ignored).

The high-level code for a non deterministic SGA based on expansion steps is listed in Fig.4, where $\mathrm{TODO}_{0}$ are the $G$-goals to be solved under theory $\langle$ thAx, $\mathscr{T}\rangle$ and generation requirements $\mathscr{G}$. The SGA is a general schema, whose core is the implementation of the expansion steps, predicates error $(S)$ and globalError $(S)$. The latter are based on the ideas presented in Section 3.1. They use the integrity constraints false :- B to detect inconsistency and store in the variable $U C$ the "unsolved constraints". To ensure the correctness of SG, an implementation has to guarantee properties $\mathrm{p} 1, \mathrm{p} 2$, p3 of expansion steps as well as the following requirements:

(i) When new objects or new witnesses (for exi) are generated in an expansion step, they are chosen according to the generation requirements, in such a way that $\mathrm{INFO}_{S} \models \mathscr{G}$ for every generated state $S$.

(ii) When error $(S)$ returns "true", then $\operatorname{INFO}_{S^{\prime}}$ is inconsistent w.r.t. $\mathscr{T}$ for every $S^{\prime}$ such that $S \preceq S^{\prime}$ ( $S$ included).

(iii) If globalError $(S)$ returns "true", then $\operatorname{INFO}_{S}$ is inconsistent with respect to $\mathscr{T}$. If it returns "false", then either $U C$ is empty and $\operatorname{INFO}_{S} \cup \mathscr{T}$ is consistent or $\mathrm{INFO}_{S} \cup \mathscr{T} \cup U C$ is inconsistent.

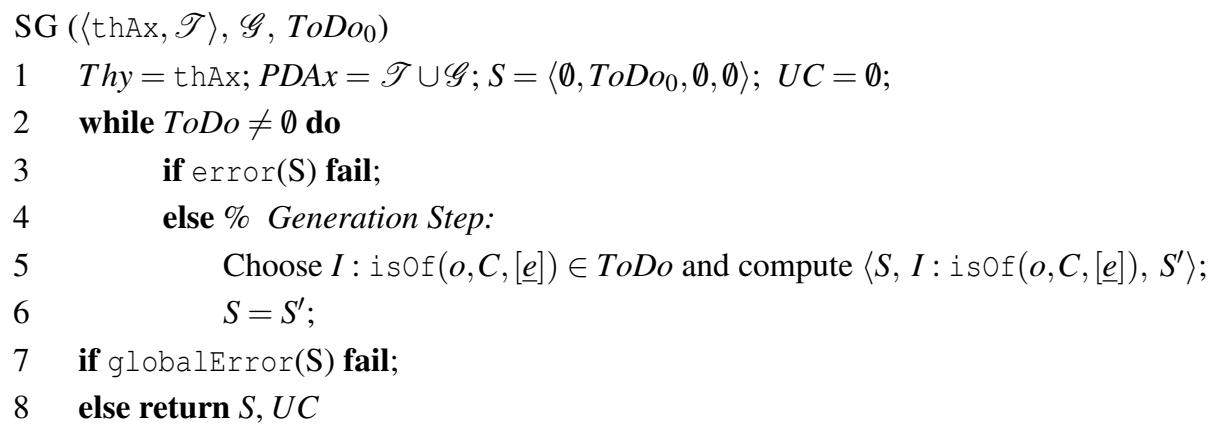

Fig. 4. The SG Algorithm

The current implementation is essentially based on a refinement of the metainterpreter considered in Section 3.1. It could be improved, namely in detecting more than trivial inconsistencies; indeed, no constraint simplification is supported.

To state the adequacy results, we introduce some additional notation (ITP) in order to associate a class $C_{j}$ and population $P$ with their information terms:

$$
\begin{aligned}
\operatorname{ITP}\left(P, C_{j}\right) & =\left[\left[\left[o_{j_{1}}, \underline{e}_{j_{1}}\right], I_{j_{1}}\right], \ldots,\left[\left[o_{j_{k}}, \underline{e}_{j_{k}}\right], I_{j_{k}}\right]\right] \\
\operatorname{ITP}(P) & =\left[\operatorname{ITP}\left(P, C_{1}\right), \ldots, \operatorname{ITP}\left(P, C_{n}\right)\right]
\end{aligned}
$$


where, $I_{j_{1}}: \operatorname{isOf}\left(o_{j_{1}}, C_{j},\left[\underline{e}_{j_{1}}\right]\right), \ldots, I_{j_{k}}: \operatorname{isOf}\left(o_{j_{k}}, C_{j},\left[\underline{e}_{j_{k}}\right]\right)$ are the $G$-goals of $P$ with class $C_{j}(1 \leq j \leq n)$; if no $G$-goal with class $C_{j}$ belongs to $P$, then $\operatorname{ITP}\left(P, C_{j}\right)$ is the empty list.

Theorem 4 (Correctness). Let $S^{*}=\left\langle\mathrm{DONE}^{*}, \emptyset, \mathrm{ClOSED}^{*}, \mathrm{INFO}^{*}\right\rangle$ be a state computed by $S G$ with theory $T=\langle t h A x, \mathscr{T}\rangle$ and generation requirements $\mathscr{G}$, and let $I^{*}=\operatorname{ITP}\left(\right.$ DONE $\left.^{*}\right)$ be the information term of the population DonE* ${ }^{*}$. Then, either UC is empty and $I^{*}:$ thAx is $\mathscr{G} \cup \mathscr{T}$-consistent, or $I^{*}:$ thAx is inconsistent with respect to $\mathscr{G} \cup \mathscr{T} \cup U C$.

The proof follows from properties (i), (ii) and (iii).

Theorem 5 (Completeness). Let $S_{0}=\left\langle\emptyset, \mathrm{ToDO}_{0}, \emptyset, \emptyset\right\rangle$ be an initial state of $S G$ with theory $T$ and generation requirements $\mathscr{G}$. If there is a state $S=\langle$ Done, $\emptyset$, CLOSED, INFO $\rangle$ such that $S_{0} \preceq S$, then the $S G A$ reaches a state $S^{*}$ in solved form such that $S_{0} \preceq S^{*} \preceq S$.

The proof follows from properties $\mathrm{p} 1, \mathrm{p} 2$ and $\mathrm{p} 3$.

\section{Related Work and Conclusion}

We have presented some features of the object-oriented modeling language CooML, a language in the spirit of UML, but based on a constructive semantics, in particular the BHK explanation of logical correctives. We have introduced a proof-theoretic notion of snapshot based on populations of objects and information terms, from which snapshot generation algorithms can be designed. More technically, we have introduced generation goals and the notion of minimal solution of such goals in the setting of a CooML specification, and we have outlined a non-deterministic generation algorithm, showing how finite minimal solutions can be, in principle, generated. We use a constraint language in order to specify the general properties of the problem domain, as well as the generation requirements. In an implementation of the SGA we assume a consistency checking algorithm, which either establishes the (in)consistency of the current snapshot, or residuates a set of unsolved constraints.

The relevance of SG for validation and testing in OO software development is widely acknowledged. The USE tool [8] for validation of UML/OCL models has been recently extended with a SG mechanism; differently from us, this is achieved via a procedural language. Other animation tools [4] are based on JML specification. In [2] the specification of features models are translated into SAT problems; tentative solutions are then propagated with a Truth Maintenance System. If a inconsistency is discovered the TMS explains the causes in view of possible model repair. Related work includes also [16], where design space specs are seen as trees whose nodes are constrained by OCL statements and BDD's are used to find solutions.

Snapshot generation is only one of CooML's aspects, once we put our software engineering glasses on and see it more generally as a specification rather than modeling language [12,9]. In this paper we have not considered methods, although the underlying logic supports a clean notion of (correct) query methods, namely those that do not 
update the system state, but extract pieces of information from it. The existence of a method $M$ answering $P$ (i.e., computing $I: P$ ) is guaranteed when $P$ is a constructive logical consequence of thAx. Moreover, $M$ can be extracted from a constructive proof of $P$. The implementation of query and update methods is a crucial part of future work.

We also plan to improve and extend the snapshot generation algorithm. There are two directions that we can pursue; first, we can fully embrace CLP as a PD logic, strengthening the connection that we have only scratched in Section 3.1. In the current prototype there is little emphasis on the simplification of unsolved constraints. This could be partially ameliorated by adopting CLP, in particular over finite domains. More in general, it is desirable to relate Theorem 3 with the notion of satisfactioncompleteness in constraint systems [7]. Another direction comes from the relation between CooML's approach to incomplete information and answer set programming [1, 17], in particular disjunctive LP [13]. A naive extension of the SGA to this case would yield inefficient solutions, yet the literature offers several ways constraints and ASP may interact [14,5]. We may explore the possibility of combining snapshot generation with SAT provers, to which we may pass ground unsolved constraints in order to check global consistency. Finally we intend to explore the more general issue of the relationships between information terms and stable models, in particular partial stable models [21]; some initial results are presented in [20].

\section{References}

1. Baral, C.: Knowledge Representation, Reasoning and Declarative Problem Solving. In: CUP (2003)

2. Batory, D.S.: Feature models, grammars, and propositional formulas. In: Obbink, H., Pohl, K. (eds.) SPLC 2005. LNCS, vol. 3714, pp. 7-20. Springer, Heidelberg (2005)

3. Boronat, A., Oriente, J., Gómez, A., Ramos, I., Carsí, J.A.: An algebraic specification of generic OCL queries within the Eclipse modeling framework. In: Rensink, A., Warmer, J. (eds.) ECMDA-FA 2006. LNCS, vol. 4066, pp. 316-330. Springer, Heidelberg (2006)

4. Bouquet, F., Dadeau, F., Legeard, B., Utting, M.: JML-testing-tools: A symbolic animator for JML specifications using CLP. In: Halbwachs, N., Zuck, L.D. (eds.) TACAS 2005. LNCS, vol. 3440, pp. 551-556. Springer, Heidelberg (2005)

5. Buccafurri, F., et al.: Strong and weak constraints in disjunctive Datalog. In: Dix et al. [6]. pp. 2-17.

6. Dix, J., Furbach, U., Nerode, A. (eds.): LPNMR 1997. LNCS, vol. 1265. Springer, Heidelberg (1997)

7. Fruewirth, T., Abdennadher, S.: Essentials of Constraint Programming. Springer, New York (2003)

8. Gogolla, M., Bohling, J., Richters, M.: Validating UML and OCL models in USE by automatic snapshot generation. Software and System Modeling 4(4), 386-398 (2005)

9. Guttag, J.V., Horning, J.J.: Larch: languages and tools for formal specification. Springer, New York, Inc., New York, NY, USA (1993)

10. Jackson, D., Wing, J.: Lightweight formal method. IEEE Computer, Los Alamitos (1996)

11. Larman, C.: Applying UML and Patterns: An Introduction to Object-Oriented Analysis and Design and Iterative Development. Prentice Hall, Upper Saddle River, NJ (2004)

12. Leavens, G.T., Baker, A.L., Ruby, C.: Preliminary design of JML: A behavioral interface specification language for Java. SIGSOFT Softw. Eng. Notes 31(3), 1-38 (2006) 
13. Leone, N., et al.: The DLV system for knowledge representation and reasoning. ACM Trans. Comput. Log. 7(3), 499-562 (2006)

14. Marek, V.W., et al.: Logic programs with monotone cardinality atoms. In: Lifschitz, V., Niemelä, I. (eds.) LPNMR 2004. LNCS (LNAI), vol. 2923, pp. 154-166. Springer, Heidelberg (2003)

15. Miglioli, P., Moscato, U., Ornaghi, M., Usberti, G.: A constructivism based on classical truth. Notre Dame Journal of Formal Logic 30(1), 67-90 (1989)

16. Neema, S., et al.: Constraint-based design-space exploration and model synthesis. In: Alur, R., Lee, I. (eds.) EMSOFT 2003. LNCS, vol. 2855, pp. 290-305. Springer, Heidelberg (2003)

17. Niemelä, I., Simons, P.: Smodels - an implementation of the stable model and well-founded semantics for normal lp. In: Dix et al. [6]. pp. 421-430

18. Offutt, J., Abdurazik, A.: Generating tests from UML specifications. In: France, R.B., Rumpe, B. (eds.) UML 1999. LNCS, vol. 1723, pp. 416-429. Springer, Heidelberg (1999)

19. Ornaghi, M., Benini, M., Ferrari, M., Fiorentini, C., Momigliano, A.: A constructive object oriented modeling language for information systems. ENTCS 153(1), 67-90 (2006)

20. Ornaghi, M., Fiorentini, C.: Answer set semantics vs. information term semantics. In: Informal Proceedings of ASP 2007: Answer Set Programming: Advances in Theory and Implementation, http://cooml.dsi.unimi.it/papers/asp.pdf

21. Przymusinski, T.C.: Well-founded and stationary models of logic programs. Ann. Math. Artif. Intell. 12(3-4), 141-187 (1994)

22. Troelstra, A.S.: From constructivism to computer science. TCS 211(1-2), 233-252 (1999)

23. Warmer, J., Kleppe, A.: The Object Constraint Language: Precise Modelling with UML. In: Object Technology Series, Addison-Wesley, Reading/MA (1999) 